$\operatorname{IPPP} / 02 / 43$

$\mathrm{DCPT} / 02 / 86$

27th September, 2002

\title{
A Z-Monitor to Calibrate Higgs Production via Vector Boson Fusion with Rapidity Gaps at the LHC
}

\author{
V. A. Khoze ${ }^{1}$, M. G. Ryskin ${ }^{2}$, W. J. Stirling ${ }^{1}$ and P. H. Williams ${ }^{1}$ \\ ${ }^{1}$ Institute for Particle Physics Phenomenology \\ University of Durham \\ Durham, DH1 3LE, U.K. \\ ${ }^{2}$ Petersburg Nuclear Physics Institute \\ Gatchina, St. Petersburg, 188300, Russia
}

\begin{abstract}
We study central $Z$-boson production accompanied by rapidity gaps on either side as a way to gauge Higgs weak boson fusion production at the LHC. We analyse the possible backgrounds for the $b \bar{b}$-decay mode and show that these can be substantially reduced. Special attention is paid to the evaluation of the gap survival factor, which is the major source of theoretical uncertainty in the rate of $H, Z$ and $W$ central production events with rapidity gaps.
\end{abstract}




\section{Introduction}

Hunting the Higgs boson(s) is the highest priority of the international high-energy physics programme. The Standard Model-like Higgs boson should have a mass between the LEP2 limit of $114 \mathrm{GeV}$ and the upper bound of about $200 \mathrm{GeV}$, which is favoured by electroweak data [1]. Within the MSSM, the light scalar Higgs boson is expected to be lighter than about $135 \mathrm{GeV}$, see for example [2]. The focus now is on searching for the Higgs at present and forthcoming hadron colliders, namely the Tevatron and the LHC.

To ascertain whether a Higgs signal can be seen, it is crucial to show that the background does not overwhelm the signal. For instance, the major difficulty in observing inclusive production of the Higgs in the preferred mass range around $115 \mathrm{GeV}$ via the dominant $H \rightarrow b \bar{b}$ mode is the huge $b \bar{b}$ QCD background. In order to rescue the $b \bar{b}$ Higgs signal different options have been proposed in the literature. An attractive possibility to reduce the background is to study the central production of the Higgs in events with a large rapidity gap on either side, see for example [3-14]. An obvious advantage of the rapidity gap approach is the clean experimental signature - hadron free zones between the remnants of the incoming protons and the Higgs decay products. The cleanest situation is in the double-diffractive exclusive process:

$$
p \stackrel{(-)}{p} \rightarrow p+H+\stackrel{(-)}{p}
$$

where the plus sign denotes a large rapidity gap. However the cross section is expected to be rather small [9, 15], and as a consequence, the corresponding event rate appears to be too low at the Tevatron. Only at the LHC is there a chance of observing this exclusive Higgs production process [11, 15, 16, [17]. Various effects cause a drastic reduction of the cross section for process (1.1), for details see [9, 18]. First, the proton form factors strongly limit the available phase space in the transverse momentum of the produced Higgs, $q_{T} \sim 1 / R_{p}$, where $R_{p}$ is the proton radius. Secondly, we have to account for the probability $\hat{S}^{2}$ that the gaps survive the soft rescattering effects of spectator partons which may populate the gaps with secondary particles, see for example [5, 19, 20]. Thirdly, the cross section is also suppressed by QCD Sudakov-like radiative effects [8, 9, 21].

The cross section is larger in the semi-inclusive case when the protons may dissociate,

$$
\left.p^{(-)}\right) \rightarrow X+H+Y
$$

but the Higgs is still isolated by rapidity gaps. In this case there is no proton form factor suppression and the QCD "radiation damage" becomes weaker. Moreover, a significant contribution to process (1.2) comes from Higgs production via $W W / Z Z$ fusion, i.e. $q q \rightarrow$ 
$q q H$. Since this process is mediated by colourless $t$-channel $W / Z$ exchanges there is no corresponding gluon bremsstrahlung in the central region [3, 4, 5, 7], and thus Sudakov suppression of the rapidity gaps does not occur. Another characteristic feature of the vector boson fusion Higgs production process is that it is accompanied by energetic quark jets in the forward and backward directions. Recently, interest in this type of Higgs production process at the LHC has risen rapidly, see for example [18, 19, 22, 23]. The particular importance of the electroweak fusion process is that it allows a determination of the Higgs coupling to vector bosons. It is worthwhile to note that the $W W / Z Z$ fusion mechanism can provide a potential way to identify $H \rightarrow b \bar{b}$ decays at the LHC, if particular kinematic configurations with large rapidity gaps are selected, see for example [18.

The most delicate issue in calculating the cross section for processes with rapidity gaps concerns the soft survival factor円 $\hat{S}^{2}$. This factor has been calculated in a number of models for various rapidity gap processes, see for example [19, 20, 24, 25]. Although there is reasonable agreement between these model expectations, it is always difficult to guarantee the precision of predictions which rely on soft physics.

Fortunately, the calculations of $\hat{S}^{2}$ can be checked experimentally by computing the event rate for a suitable calibrating reaction and comparing with the observed rate. As shown in [9, 21] the appropriate monitoring process for the double-diffractive mechanism is central dijet production with a rapidity gap on either side. To date, such a check has been the prediction of diffractive dijet production at the Tevatron in terms of the diffractive structure functions measured at HERA [26]. The evaluation of the survival factor $\hat{S}^{2}$ based on the formalism of [19, 20] appears to be in remarkable agreement with the CDF data (see also [27, 28]). We expect that future measurements in run II of the Tevatron will provide us with further detailed information on $\hat{S}^{2}$.

As was pointed out in [29, 30], the survival factor for the gaps surrounding $W W \rightarrow H$ fusion can be monitored experimentally by observing the closely related central production of a $Z / W$ boson with the same rapidity gap and jet configuration. The discussion in 29, 30] concerns the studies of $Z / W+2$ forward jet production with subsequent leptonic $Z$ decay in association with a rapidity gap trigger, which could allow a substantial suppression of the QCD-induced backgrounds. It is worthwhile to keep in mind that in different papers different criteria are used for the definition of the rapidity gaps. For example, in the approach of [23, 30] no jets with $p_{T}>10-20 \mathrm{GeV}$ are permitted within the gaps, while in [8, 9, 11] the gap is required to be completely devoid of any soft hadrons.

\footnotetext{
${ }^{1}$ Recall that this factor is not universal, but is very sensitive to the spatial distribution of partons inside the colliding protons, which in turn results in the dependence on the particular hard subprocess as well as on the kinematical configurations of the parent reaction 19,20 .
} 
Note that the determination of the gap survival factor in the vector boson mediated process is interesting in its own right, since here we can separate the contribution of the short transverse size component of the proton [20], which so far has not attracted much attention, either theoretically or experimentally.

The reader should be warned about the potential problems with the identification of rapidity gaps in the real life experimental environment at the LHC. When the LHC operates at medium and high luminosity, the recorded events will be plagued by overlap interactions in the same bunch crossing (pile-up). However, as discussed in [17], at least at the medium luminosity of $10^{33} \mathrm{~cm}^{-2} \mathrm{~s}^{-1}$ the gaps should be detectable. Using vertex reconstruction information, one can separate particles originating from the same vertex as the high- $E_{T}$ jets from those relatively low- $p_{T}$ particles which arise from other vertices corresponding to the pile-up interactions. However, it is quite unlikely that this technique can be used for the super-LHC luminosity of $10^{35} \mathrm{~cm}^{-2} \mathrm{~s}^{-1}$.

In this paper we develop the ideas of [29, 30] further by considering the decays of both (light) Higgs and $Z$ bosons into $b \bar{b}$ pairs, the dominant decay channel of the former 2 . In each case we require two forward energetic jets, and rapidity gaps on either side of the centrally produced decay products. Both $H$ and $Z$ can be produced by electroweak vector boson fusion, for which gaps are 'natural', but the $Z$ can also be produced via $\mathscr{O}\left(\alpha_{S}^{2}\right)$ QCD processes, with both quarks and gluons exchanged in the $t$-channel, but as we shall see these have a smaller soft survival factor. Finally, there is a potentially large continuum $b \bar{b}$ background, which is again heavily suppressed when rapidity gaps are required.

The paper is organised as follows. In Section 2 we discuss the calculation of the various signal and background processes at the parton level. In Section 3 we perform detailed numerical calculations with realistic experimental cuts to determine the corresponding cross sections. In Section 4 we explore the consequences of hadronisation and estimate the gap survival probabilities, $\hat{S}^{2}$, for the various processes under consideration. Finally, in Section 5 we combine the parton-level cross sections with the gap survival probabilities to give our final predictions for the cross sections. Section 6 summarises our conclusions.

\section{Parton Level Calculation of Higgs, $Z$ and $W$ pro- duction}

In this section we assemble the various parton-level cross sections that are used in our analysis. We are particularly interested in the overall event rates for the various signal

\footnotetext{
${ }^{2}$ For completeness, we also consider the $W+2$ jet production process.
} 
and background processes, and in the kinematic distributions of the final-state particles. All matrix elements used in the cross-section calculations are obtained using MADGRAPH [31]. In all cases we work in the zero width approximation for the centrallyproduced bosons.

We begin by considering the fundamental signal process, $\mathscr{O}\left(\alpha_{W}^{3}\right)$ Higgs production by $W W, Z Z$ fusion: $q q \rightarrow q q H$ (Fig. 1). We assume that the Higgs is light, so that the dominant decay is into the $b \bar{b}$ final state. Because the momentum transfer is much smaller than the energy of the struck quark jets $\left(\left\langle p_{T}\right\rangle \sim M_{W / Z}\right)$, the jets are produced predominantly at small angle (i.e. large rapidity). Note that there is no exchange of colour in the $t$-channel, which leads to a suppression of hadronic radiation in the central region between the forward jets [3, 4, 5, 7].

Representative Feynman diagrams for the analogous $\mathscr{O}\left(\alpha_{W}^{3}\right) Z$ production process, $q q \rightarrow q q Z$ and $q \bar{q} \rightarrow q \bar{q} Z$ are shown in Fig. 2. They were first analysed in [29, 30]. Note that in addition to the $W W$ fusion diagram, Fig. 2(a), the $Z$ can also be radiated off either of the incoming or outgoing quark lines, Figs. E(b) and (c). The characteristic topology of (b) is of a $Z$ preferentially produced in the forward or backward region close in rapidity to one of the final-state quark jets. Requiring centrally produced $Z$ decay products tends to suppress this contribution. Process (c) corresponds to $s$-channel production of the final-state $q \bar{q}$ pair, with the $Z$ boson emitted off the incoming quark lines. It does not correspond to $t$-channel colour singlet exchange and is heavily kinematically suppressed by requiring a large rapidity separation between the jets.

Similar remarks apply to $W$ production. Representative Feynman diagrams for $q q \rightarrow$ $W q q$ are shown in Fig. 3. Note that the central $W$-production via $\gamma$ exchange corresponding to Fig. 3a was recently discussed in 32].

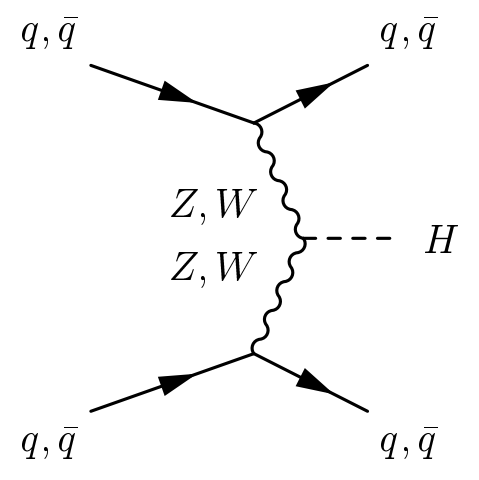

Figure 1: Higgs production via electroweak vector boson fusion.

The above $\mathscr{O}\left(\alpha_{W}^{3}\right) H$ and $Z$ production processes both therefore give rise to rapidity 


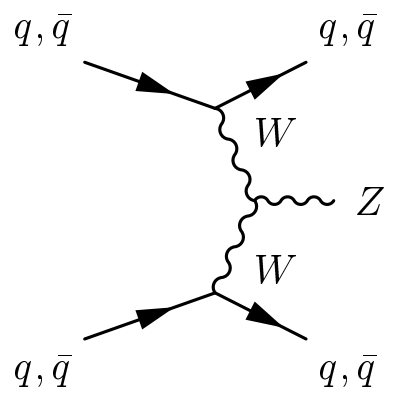

(a)

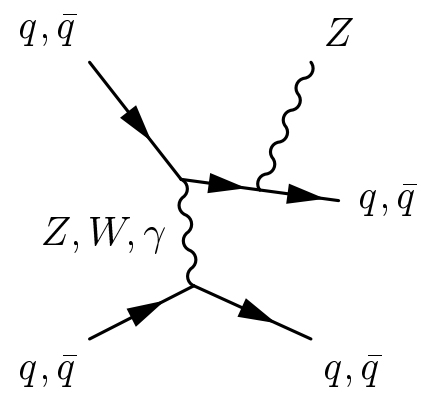

(b)

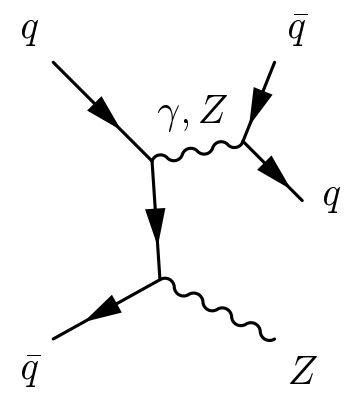

(c)

Figure 2: The three topologies for $Z q q$ production via electroweak vector boson exchanges.

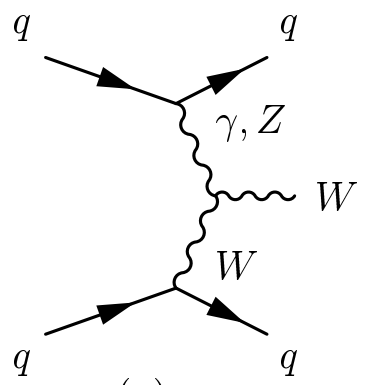

(a)

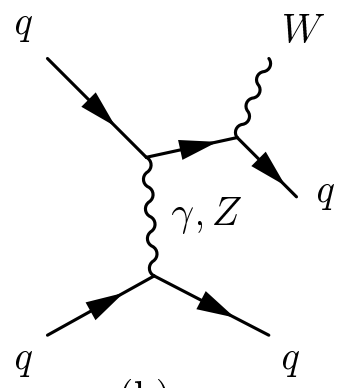

(b)

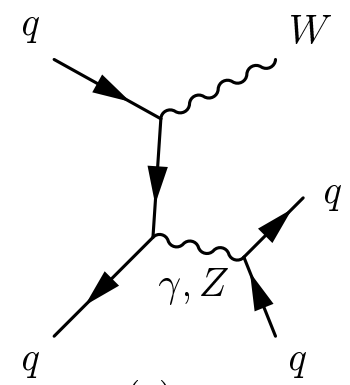

(c)

Figure 3: The three topologies for $W$ production.

gap signatures between the forward jets and the central $H$ and $Z$ decay products. However there is a potentially important QCD $\mathscr{O}\left(\alpha_{S}^{2} \alpha_{W}\right)$ background contribution to $Z+2$ jet production where the internal electroweak gauge boson is replaced by a gluon. More generally, at this order indistinguishable background contributions can arise from any $2 \rightarrow 2$ scattering process (other than $g g \rightarrow g g$ ) where the $Z$ is radiated off a quark line. Representative Feynman diagrams are shown in Fig. ․ By selecting forward jets and central $Z$ bosons, in order to mimic the dominant Higgs configuration, the $t$-channel momentum transfer is minimised, and these QCD processes split into two types: $t$-channel quark (Figs. $4(\mathrm{a}, \mathrm{b}, \mathrm{c}))$ and gluon exchange (Figs. $4(\mathrm{~d})$ ). Requiring rapidity gaps therefore suppresses both type of contribution, as will be discussed in Section 4 below.

Finally, given that we are interested in the $b \bar{b}$ decay modes of both the Higgs and $Z$ bosons, with two additional jets in the final state, there is a class of $\mathscr{O}\left(\alpha_{S}^{4}\right)$ pure-QCD background processes of the form $a b \rightarrow c d+b \bar{b}$ with $a \ldots d=q, g$, examples of which are shown in Fig. 5. We will consider the corresponding cross sections in the following section, with the additional requirement that $m_{b \bar{b}} \simeq M_{Z}$. 


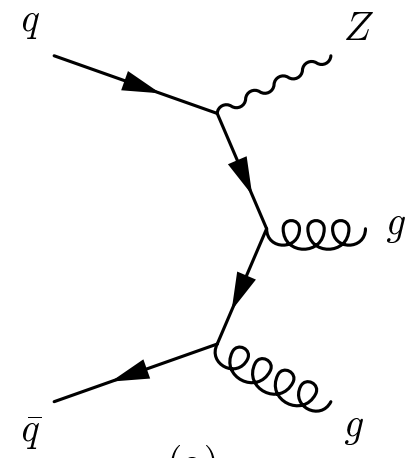

(a)

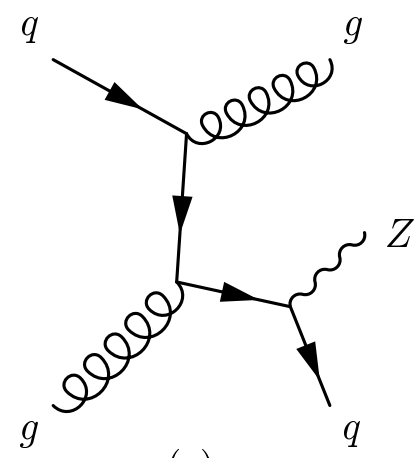

(c)

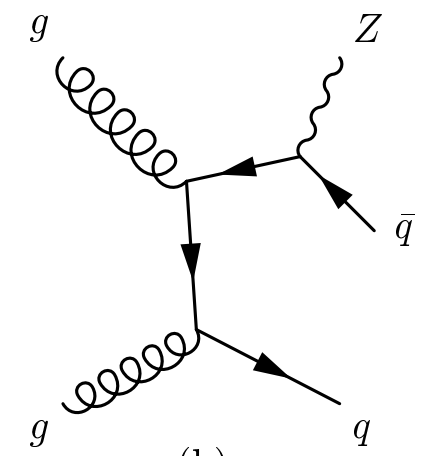

(b)

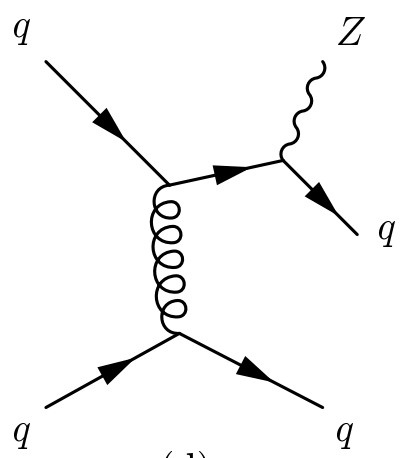

(d)

Figure 4: QCD background processes to $Z$ production.

\section{Signal and Background Rates and Properties}

In this section we calculate cross sections and kinematic distributions for the processes described in the previous section, for proton-proton collisions at the LHC using a representative set of cuts on the final state particles. We have in mind final states with a jet registered in a forward detector with pseudorapidity $\eta_{1}>\eta_{\min }$, another produced backwards with $\eta_{2}<-\eta_{\min }$, and the $H, Z$ and $W$ decay products produced centrally, with rapidity $\left|y_{H, Z, W}\right|<y_{\max }$. We impose a minimum transverse momentum cut $p_{T \min }$ on all final-state jets.

\subsection{Total cross sections}

Figure 6 shows the total cross section for Higgs, electroweak $Z$ and $W$, and QCD $Z$ production (with no branching ratios included) as a function of a cut on the minimum transverse jet momentum $p_{T \text { min }}$. The Higgs mass is $M_{H}=115 \mathrm{GeV}$ and the leading-order MRST98LO [33] parton distribution set is used. Note that only for $H$ production is the 

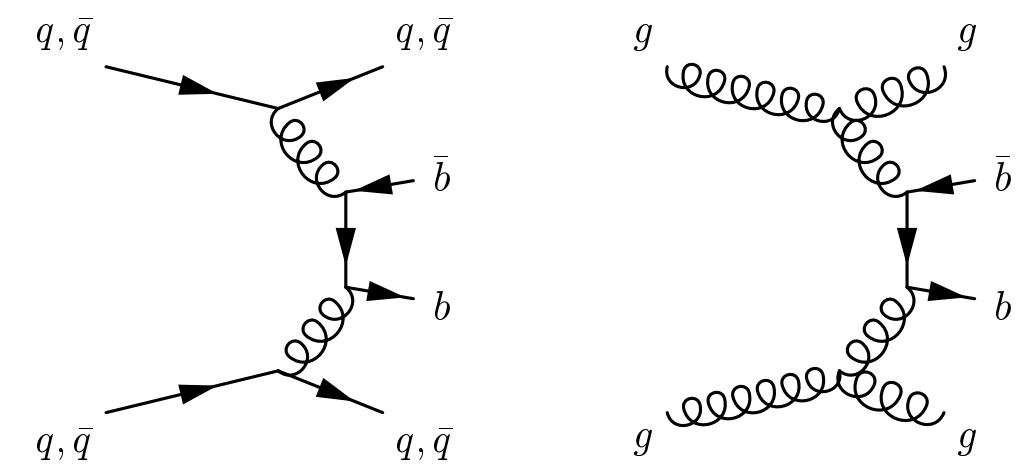

Figure 5: QCD backgrounds to $q q \rightarrow q q(H, Z),(H, Z) \rightarrow b \bar{b}$.

cross section finite in the limit $p_{T \text { min }} \rightarrow 0$. In addition, the possibility that the final state jets in $Z$ and $W$ production originate in the splitting process $g^{*}, \gamma^{*} \rightarrow q \bar{q}$ (for example, see Fig. 2(c)) requires a jet separation cut. The minimal way to do this is simply to require that one of the jets is produced in the forward hemisphere and the other in the backward hemisphere, i.e. $\eta_{1} \cdot \eta_{2}<0$. When we come to consider 'realistic' cuts, in particular to isolate the jets from each other and the $H$ and $Z$ decay products, we will impose a large rapidity separation cut in which one jet is produced far forward and one far backward: $\left|\eta_{1}\right|,\left|\eta_{2}\right|>\eta_{\min }, \eta_{1} \cdot \eta_{2}<0$. For the Higgs production process, which has no infrared or collinear singularities, the imposition of $p_{T \min }$ and $\eta_{1} \cdot \eta_{2}<0$ acceptance cuts simply reduces the cross section slightly (by approximately $25 \%$ for a broad range of $p_{T \text { min }}$ values), see Fig. 6 .

Figure 6 shows that there is a strong ordering of the cross sections $\sigma(Z, Q C D) \gg$ $\sigma(Z, E W) \gg \sigma(H)$, with $\sigma(Z, Q C D)$ exhibiting the strongest dependence on $p_{T \text { min }}$. The $W$ cross section has a stronger infra-red singularity as $p_{T \text { min }} \rightarrow 0$ than the corresponding $Z$ cross section, due to the soft photon singularity present in the extra diagram with respect to the $Z$ production process involving the triple gauge boson vertex (Fig. 3 (a)). This is shown more clearly in $W / Z$ cross section ratio plot, Fig. 7). The Higgs cross section is only weakly dependent on the mass $M_{H}$, decreasing by a factor of 2 as $M_{H}$ increases from $100 \mathrm{GeV}$ to $200 \mathrm{GeV}$, see Fig. 8.

Note that all the above cross sections are evaluated in the zero $Z / W$ width approximation and at leading order in perturbation theory. In particular, in the QCD $Z+2$ jet calculation the scale of the strong coupling $\alpha_{S}$ is not determined, and there is a nonnegligible scale dependence uncertainty as a result. We use $\alpha_{S} \equiv \alpha_{S}\left(M_{Z}^{2}\right)$ throughout.

\footnotetext{
${ }^{3}$ The possibility of exchanging a massless photon or gluon in the $t$ channel gives rise to an infrared singularity in the electroweak and QCD $Z+2$ jet production processes as $p_{T \min } \rightarrow 0$, see Figs. 2 and 1 . For exclusive $p p \rightarrow p+X+p$ collisions this singularity is cut off by the $t_{\mathrm{min}}$-effect.
} 


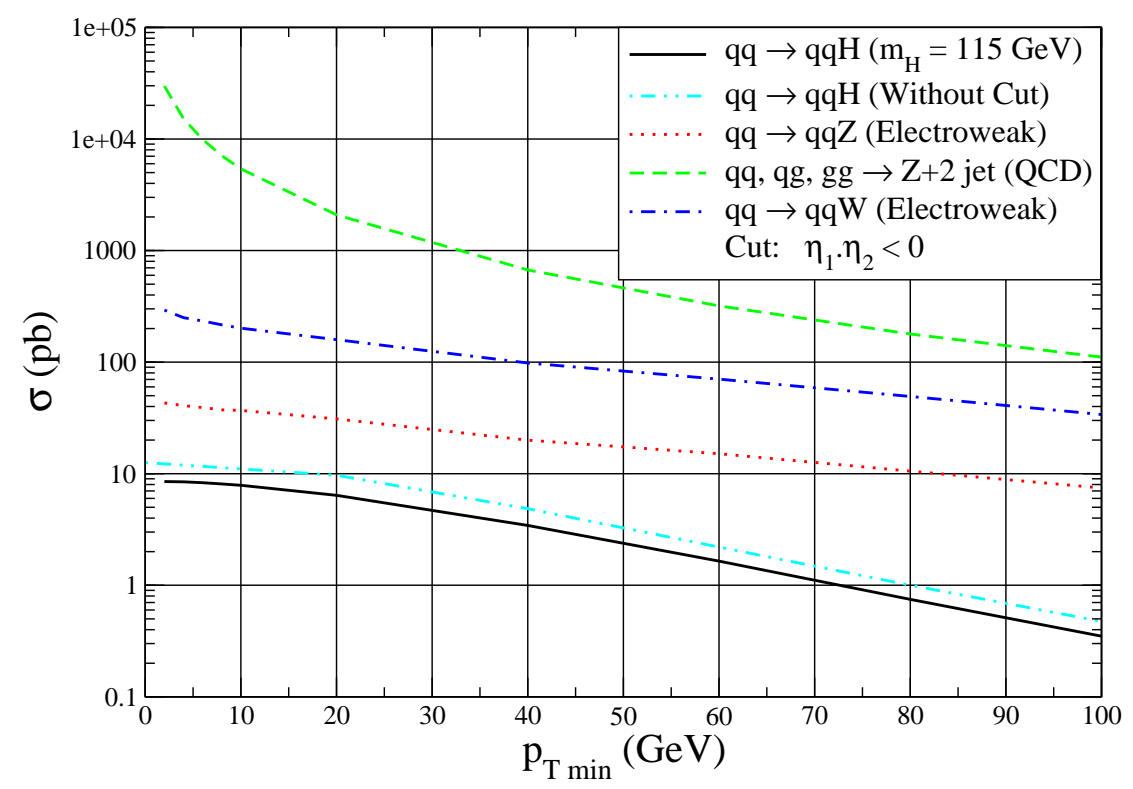

Figure 6: Total cross sections for $(H, Z, W)+2$ jet production in $p p$ collisions at $\sqrt{s}=14$ $\mathrm{TeV}$ as a function of the cut on the jet transverse momentum, $p_{T \text { min }}$. Rapidity cuts on the final state jets are also imposed, as indicated.

One could also argue for a smaller scale characteristic of the transverse momenta of the forward jets, e.g. $\alpha_{S} \equiv \alpha_{S}\left(p_{T \min }^{2}\right)$. We will discuss the impact of such a choice on our predicted event rates in Section 5 .

\subsection{Distributions}

Our objective is to find a set of selection cuts that minimises the background while not affecting drastically the Higgs, $Z$ and $W$ rapidity gap signal. We begin by calculating the transverse momentum and rapidity distributions of the jets in $q q H$ production, Figs. 9 and 10. Evidently the jets are predominantly produced with transverse momenta of order $M_{W} / 2 \sim 40 \mathrm{GeV}$, and with a rapidity separation of around 5, see Fig. 11. Notice the small excess around $\Delta \eta \sim 1 / 2$. This is caused by the contributing process $q \bar{q} \rightarrow H q \bar{q}$ in which $m_{j j} \sim M_{Z}$, i.e. the Higgs is produced in association with a $Z$ (or $W$ ) boson which subsequently decays into a $q \bar{q}$ pair, see Fig. 12. This is more clearly seen in the dijet mass distribution, Fig. 13.

Requiring the jets to be well-separated in rapidity forces $m_{j j}$ to be large and this resonant contribution is strongly suppressed. For example, Fig. 13 also shows the dijet 


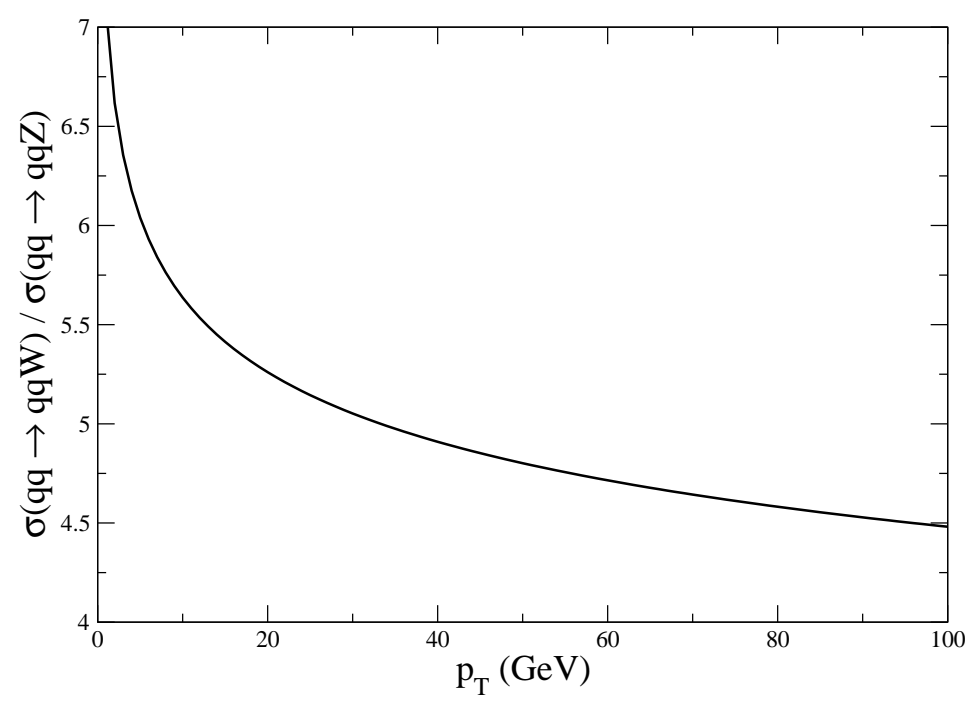

Figure 7: The ratio between the total cross sections of the $W$ and $Z$ (electroweak) production processes as defined in the previous figure. The $W$ cross section is 'more divergent' than that for $Z$ production at low $p_{T}$, because of the extra photon-exchange diagram involving the triple gauge boson vertex (Fig. $3(\mathrm{a})$ ).

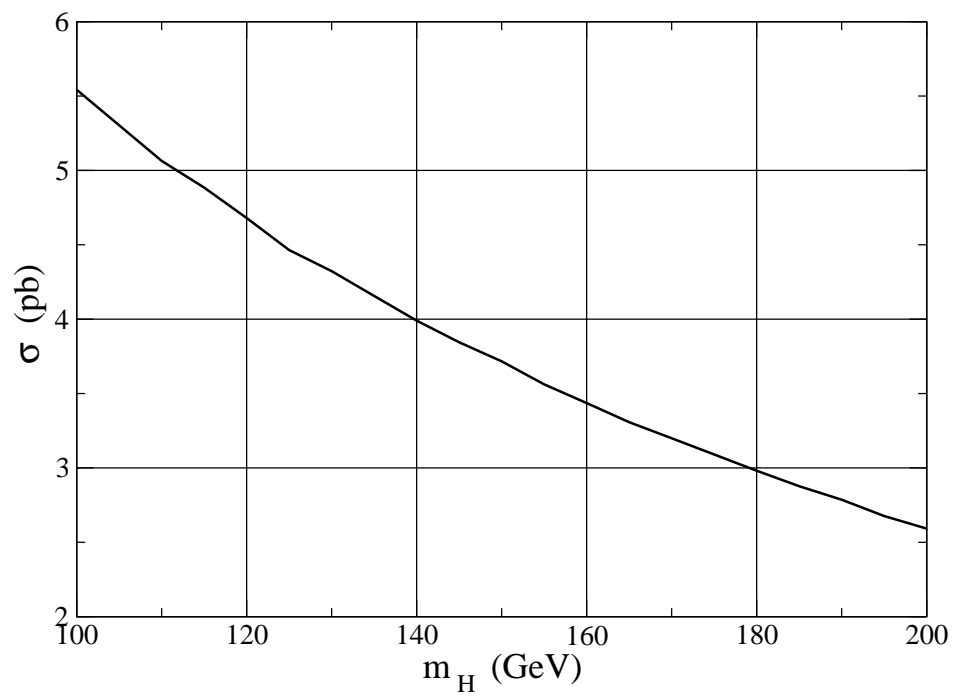

Figure 8: The $\sqrt{s}=14 \mathrm{TeV} q q \rightarrow q q H$ cross section as a function of the Higgs mass $M_{H}$. 


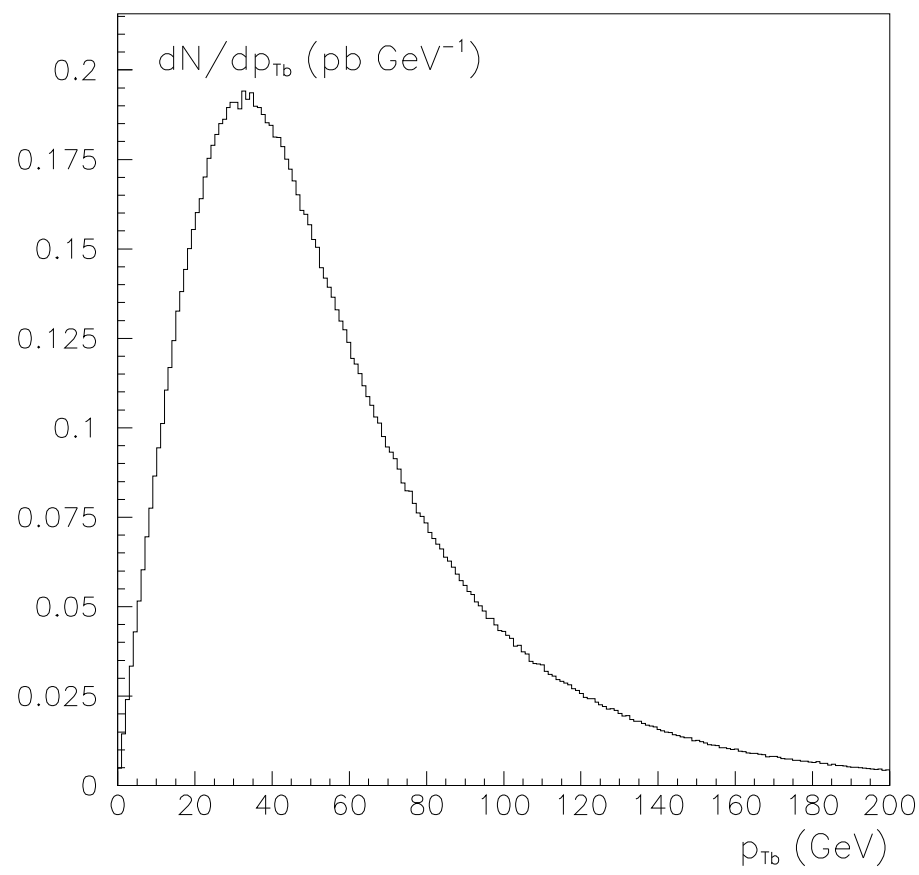

Figure 9: Jet transverse momentum distribution for $q q \rightarrow q q H$. The peak is at around $M_{W} / 2$.

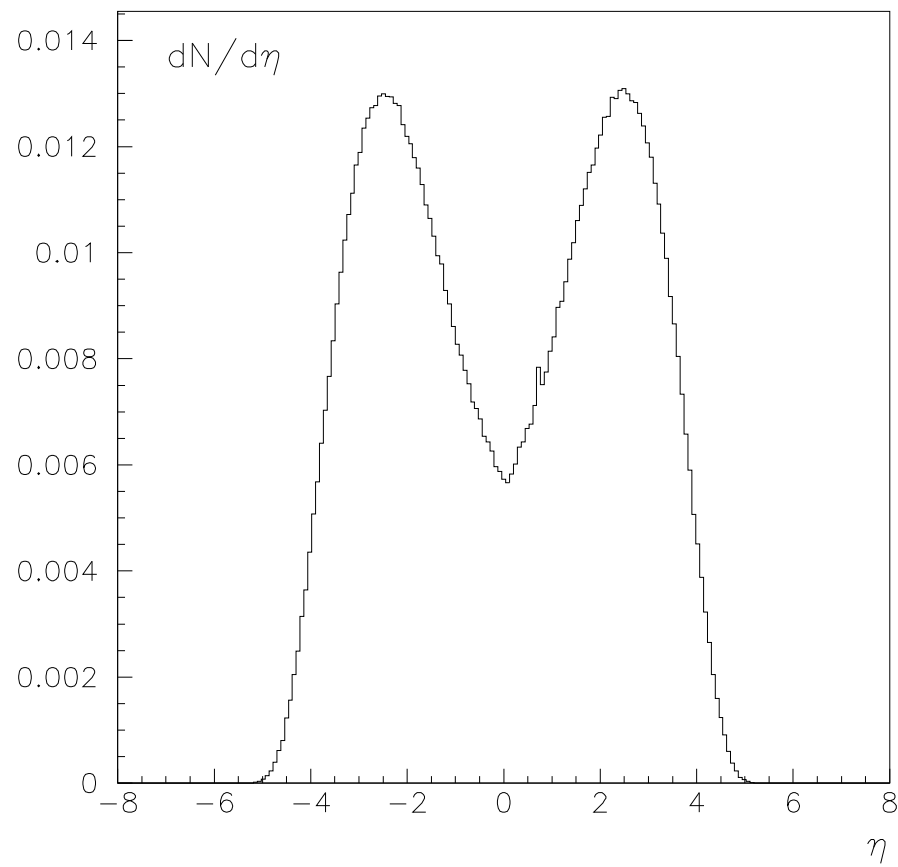

Figure 10: Rapidity distribution of jets for $q q \rightarrow q q H$ with $p_{\text {Tmin }}=40 \mathrm{GeV}$ and $\eta_{1} . \eta_{2}<0$. The vertical scale is normalised arbitrarily. 


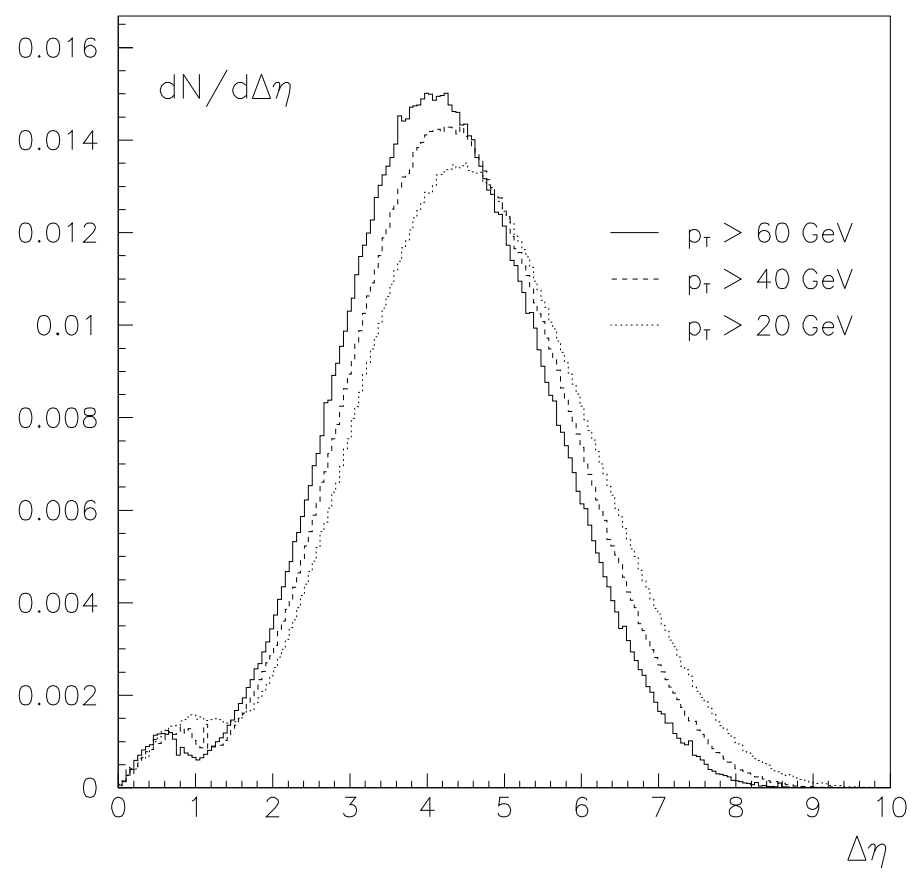

Figure 11: Dijet rapidity difference for $q q \rightarrow q q H$ (with $\eta_{1} . \eta_{2}<0$ ) as a function of the $p_{T \min }$ transverse momentum cut. The vertical scale is normalised arbitrarily. The gap narrows marginally as the cut is raised, as expected from kinematics. The small excess seen at low $\Delta \eta$ is discussed in the text.

mass distribution for $\left|\eta_{1,2}\right|>\eta_{\min }=2$.

The jet rapidity distribution for electroweak $q q Z$ production is shown in Fig. 14. Comparing with Fig. 10 for $q q H$, we see that the jets produced with a $Z$ are more uniform in rapidity, The ' $W W$-fusion' diagrams of Fig. 2 (a) still produce jets with a large separation, but the central region is now filled in by contributions from the other nonfusion ' $Z$-bremsstrahlung' processes, Figs. Z(b,c). Electroweak $W$ production has very similar characteristics to electroweak $Z$ production.

For the QCD background to electroweak $Z$ production, the jets are produced much more centrally, see Fig. 15. Requiring a jet in each forward/backward hemisphere leads to a typical rapidity separation of about 3, as shown in Fig. 16, which is significantly less than for either $H$ or electroweak $Z$ production. There is no natural rapidity gap, as for the $t$-channel colour-singlet exchange processes.

\subsection{Selection cuts}

We can now proceed to define a set of selection cuts that leads to a sample of $H, Z$ and $W$ events with the potential to exhibit rapidity gaps. Since our primary goal is to calibrate 


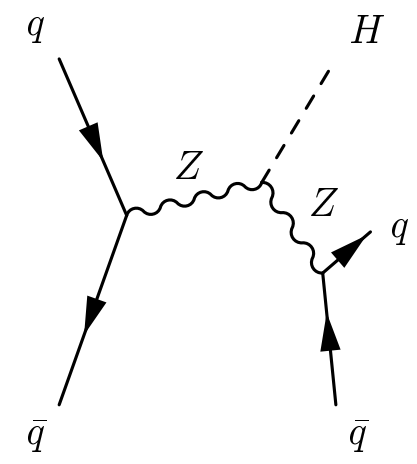

Figure 12: Contribution to the $\mathscr{O}\left(\alpha_{W}^{3}\right)$ electroweak process $q \bar{q} \rightarrow H q \bar{q}$ that resonates when $m_{j j} \sim M_{Z}$

the gap survival for Higgs production, we will concentrate first on the $b \bar{b}$ decays of $H$ and $Z$, the latter produced either via electroweak or QCD processes.

When considering the $b \bar{b}$ decay modes of both the Higgs and $Z$ bosons, we must include also the important irreducible background from QCD $\mathscr{O}\left(\alpha_{S}^{4}\right) b \bar{b}+2$ jet production, see Fig. 5. Such processes give a continuous distribution of $m_{b \bar{b}}$ masses, and in what follows we impose a cut of $\left|m_{b \bar{b}}-M_{Z}\right|<10 \mathrm{GeV}$ to select those background events that mimic $Z \rightarrow b \bar{b}$ decay.

The configuration we have in mind has one jet registered in a forward detector with $\eta>\eta_{\min }$, another produced backwards with $\eta<-\eta_{\min }$, and the two $b$ jets from $H$ and $Z$ decay produced centrally. From the results of the previous section, such a selection will in principle preserve the bulk of the Higgs signal while suppressing the (non-gap) QCD $Z$ and $b \bar{b}+2$ jet production.

For both ATLAS and CMS, the forward hadron calorimeters cover approximately $3<|\eta|<5$, and so we will require our forward dijets to be produced in this region of rapidity, with $p_{T}>p_{T \min }=40 \mathrm{GeV}$. In order to separate the $H, Z$ decay jets from the forward jets, we require $\left|\eta_{b}\right|<1.5$, and $p_{T b}>10 \mathrm{GeV}$. Although these cuts are designed to reflect the 'natural' characteristics of $q q H$ production, they do result in a non-negligible loss of signal rate, even before $b$-tagging efficiencies etc. are taken into account. This is illustrated in Table 1, which quantifies the effect on the cross section of applying the cuts sequentially. One can see that imposing forward jet cuts has the largest impact on the cross section, and indeed this is the case for all the processes considered.

Figures 17 and 18 show the cross sections at $\sqrt{s}=14 \mathrm{TeV}$ as a function of $p_{T \min }$ for

\footnotetext{
${ }^{4}$ The typical transverse momentum of the jets in both the signal and background processes is $\sim M_{Z} / 2$, and this cut does not have any significant effect on the event rates, see for example Table 1 below.
} 


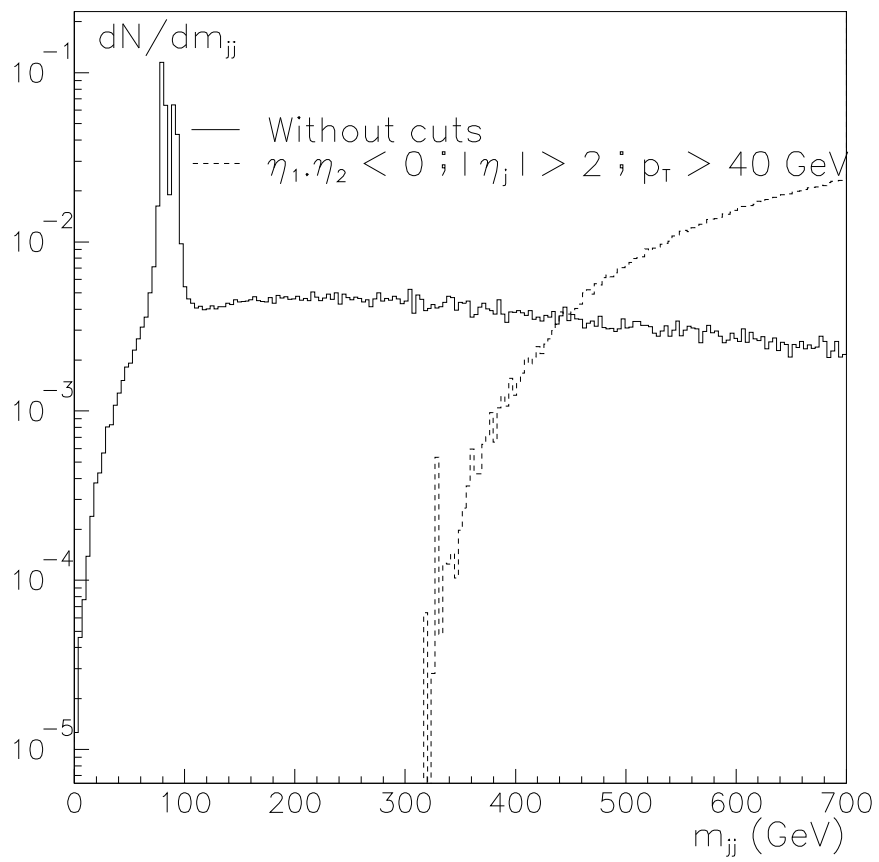

Figure 13: The dijet invariant mass for $q q \rightarrow q q H$ shows a double resonance around $m_{Z}$ and $m_{W}$ - due to diagrams such as Fig. 12. The vertical scale is normalised arbitrarily. When the jets are required to be separated and forward in rapidity the effect is irrelevant.

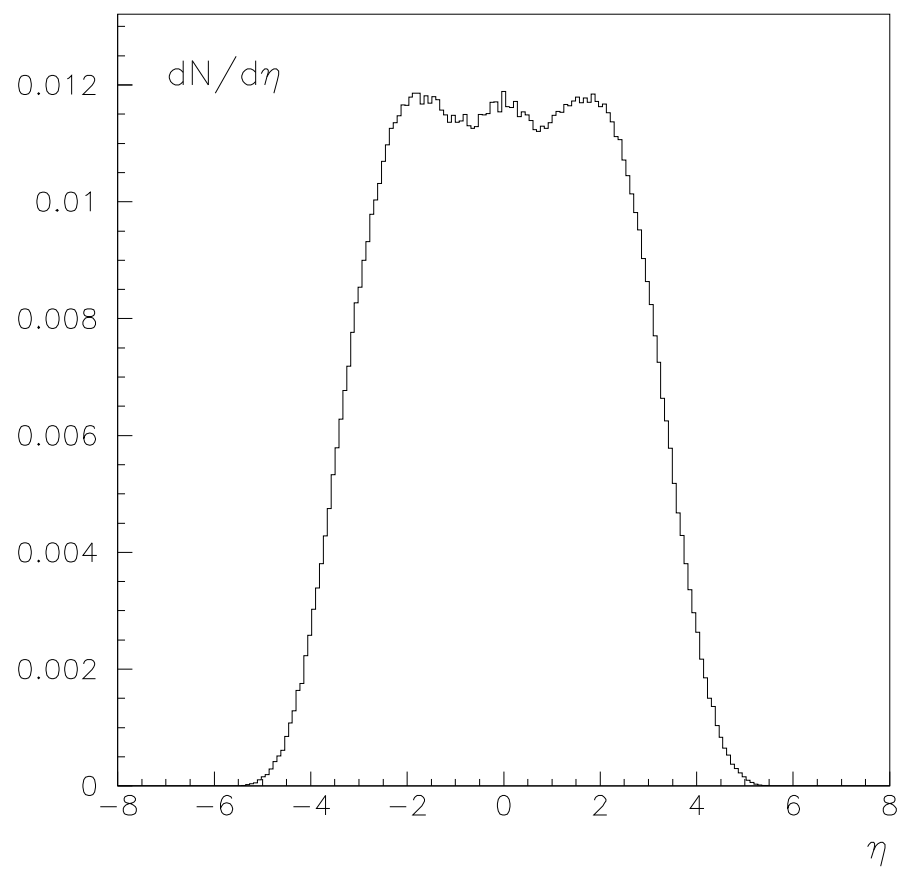

Figure 14: Rapidity distribution of jets for electroweak $Z$ production. The vertical scale is normalised arbitrarily. The gap is narrower than for the Higgs signal (Fig. 10). A jet transverse momentum cut of $p_{T \min }=40 \mathrm{GeV}$ is applied, as is $\eta_{1} . \eta_{2}<0$. 


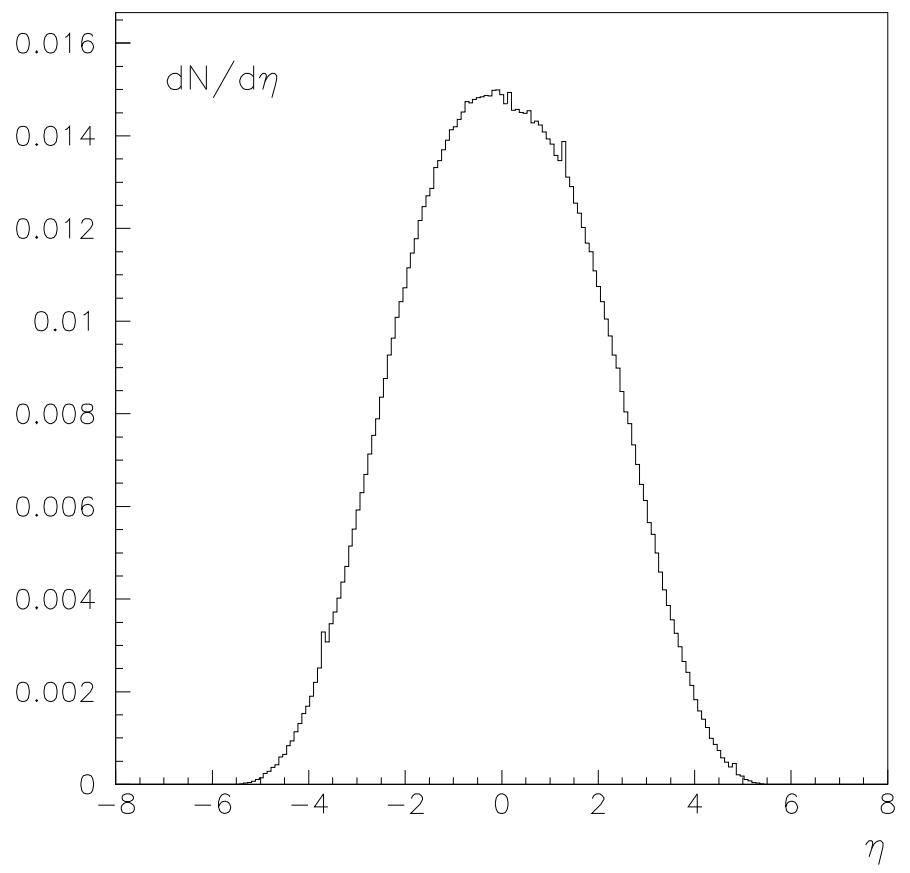

Figure 15: Rapidity distribution of jets for QCD $Z+2$ jet production. The vertical scale is normalised arbitrarily. A jet transverse momentum cut of $p_{T \min }=40 \mathrm{GeV}$ is applied, as is $\eta_{1} . \eta_{2}<0$.

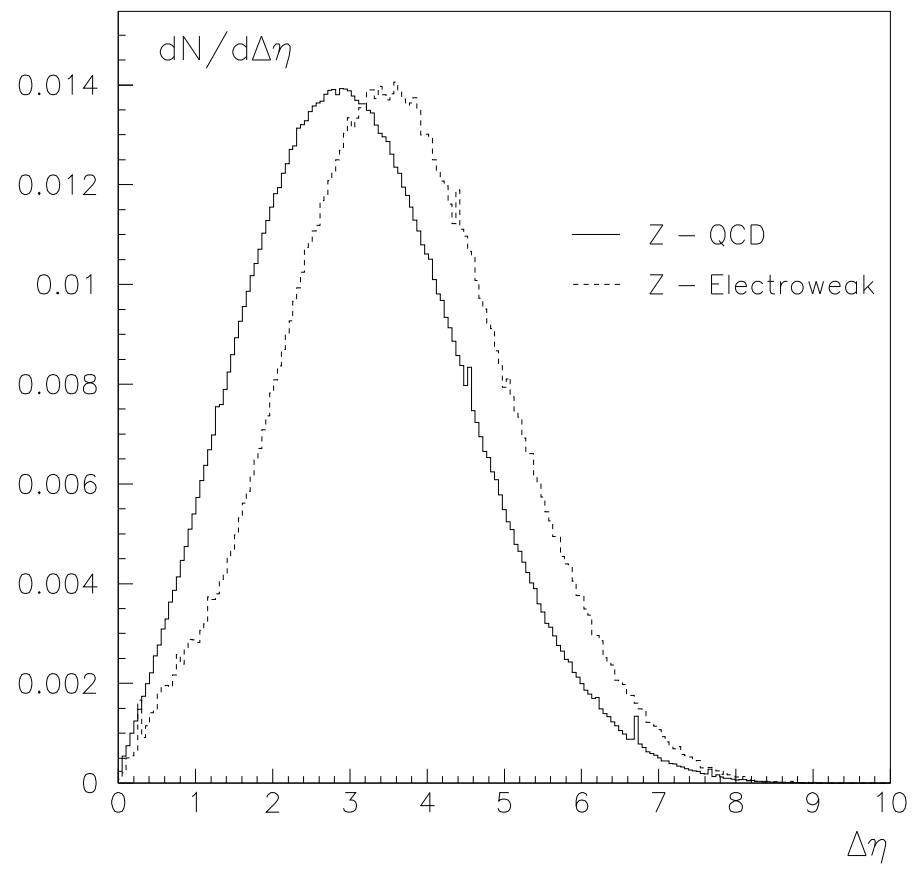

Figure 16: Comparison of the dijet rapidity differences for the electroweak and QCD $Z$ production processes. The vertical scale is normalised arbitrarily. A jet transverse momentum cut of $p_{T \text { min }}=40 \mathrm{GeV}$ is applied, as is $\eta_{1} \cdot \eta_{2}<0$. 


\begin{tabular}{|c|c|c|}
\hline Cut Imposed & Cross Section for $q q \rightarrow q q H$ at $p_{T}>40 \mathrm{GeV}$ & \% of Initial Cross Section \\
\hline \hline & $4.86 \mathrm{pb}$ & $100 \%$ \\
\hline $\operatorname{Br}(H \rightarrow b \bar{b})$ & $3.49 \mathrm{pb}$ & $71.9 \%$ \\
\hline$\eta_{1} \cdot \eta_{2}<0$ & $2.47 \mathrm{pb}$ & $50.8 \%$ \\
\hline$\Delta \eta_{j}>6$ & $0.495 \mathrm{pb}$ & $10.2 \%$ \\
\hline$\left|\eta_{j}\right|>3$ & $0.0990 \mathrm{pb}$ & $2.04 \%$ \\
\hline$\left|\eta_{b}\right|<1.5$ & $0.0465 \mathrm{pb}$ & $0.957 \%$ \\
\hline$p_{T b}>10 \mathrm{GeV}$ & $0.0463 \mathrm{pb}$ & $0.953 \%$ \\
\hline
\end{tabular}

Table 1: Loss of $q q \rightarrow q q H$ cross section at $\sqrt{s}=14 \mathrm{TeV}$ with $M_{H}=115 \mathrm{GeV}$ in applying selection cuts and the $b \bar{b}$ branching ratio.

all processes. The Higgs production cross section is reduced by a factor of $\sim 100$ and the electroweak $Z$ production by $\sim 1000$ in comparison with Figure 6 6 . The cuts reduce the $Z$ production QCD background by a factor of $\sim 10000$. As already mentioned, in evaluating the pure QCD $b \bar{b}$ production cross sections we further impose the restriction that the dijet invariant mass be within $10 \mathrm{GeV}$ of $M_{Z}$.

\section{Gap Survival Probability}

\subsection{Parton Level}

In the previous sections we have presented cross sections for Higgs, Z and QCD $b \bar{b}$ production processes for events with rapidity gaps at the parton level. We take the definition of rapidity gap to mean that there should be no minijets with a large $\left(p_{T}>10 \mathrm{GeV}\right)$ transverse momentum within the gap region. As discussed in the introduction, the selection of rapidity gap events improves the signal to background ratio because gaps are a characteristic feature of the vector boson fusion process, whereas they are not for QCD $Z$ and continuum $b \bar{b}$ production. Of course, the results presented in the previous section should be corrected to account for the rescattering of spectator partons, that is the possibility that another pair of initial, fast partons interacts inelastically in the same event. Secondaries produced in this inelastic interaction may fill the gap and the probability, $\hat{S}^{2}$, for the gap to survive depends on the criteria used to select the gap. Insofar as we

\footnotetext{
${ }^{5}$ This is because of the difference in rapidity distributions in the $H$ (Figs. 10 and 11) and $Z$ (Figs. 14 and 16) cases which is caused by the process shown in Figs 2(b),(c) and Fig. E(d) where the quark jets are closer to each other.
} 


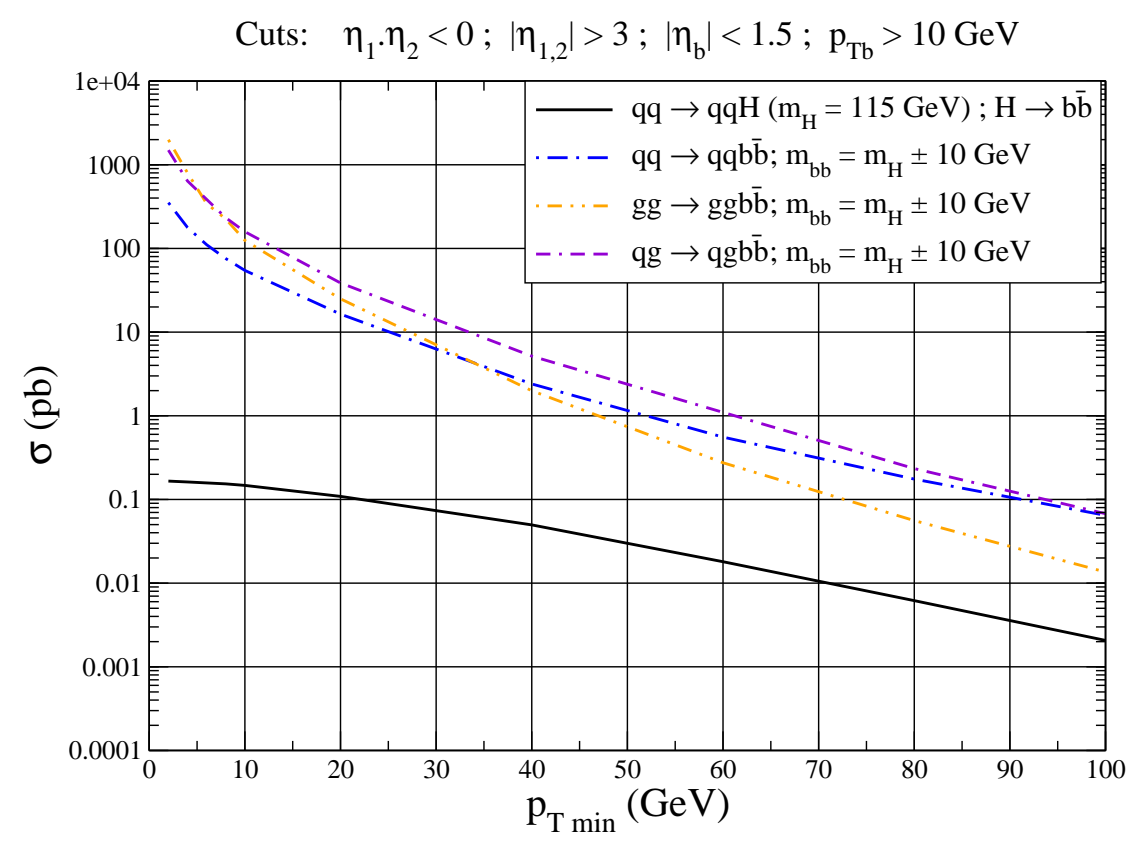

Figure 17: Cross sections at $\sqrt{s}=14 \mathrm{TeV}$ for Higgs production processes after application of the cuts described in the text.

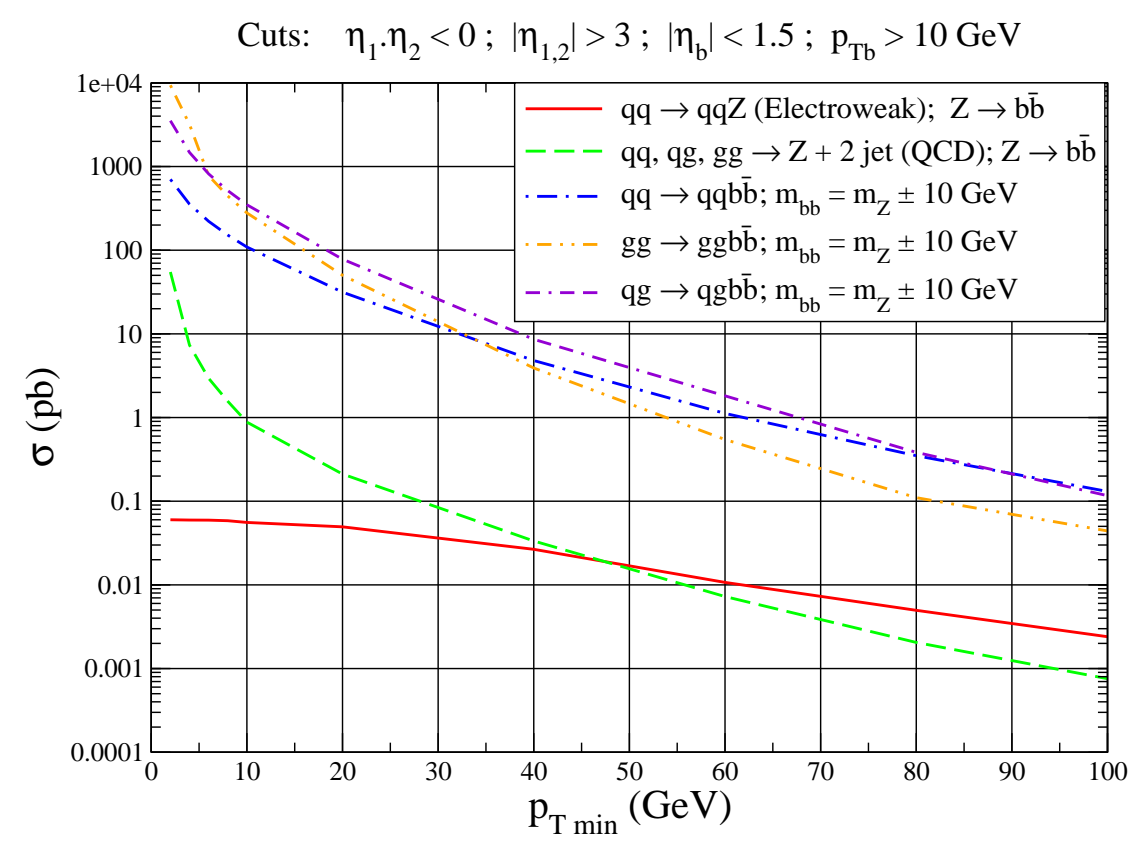

Figure 18: Cross sections at $\sqrt{s}=14 \mathrm{TeV}$ for $Z$ production processes after application of the cuts described in the text. 
require only to have no high $p_{T}$ particles (or minijets) within the gap interval, the effect is not too large. The probability to produce high $p_{T}$ secondaries is relatively small and the corresponding survival factor $\hat{S}^{2} \simeq 0.7-0.9$ (depending on the $p_{T}$ cut) is more or less close to one, see for example [23]. For any specific kinematics (and $p_{T}$ cuts), the value of $\hat{S}^{2}$ for such 'parton level' gaps may be estimated using, for example, one of the options of the PYTHIA Monte Carlo program [34], or an 'exponentiation' model such as that used in 35 .

\subsection{Hadron Level}

As seen in Figs. 17, 18, the QCD-induced $b \bar{b}$ background is still large. It exceeds by two orders of magnitude the $Z / H$ cross sections and it is therefore necessary to suppress the background further. This can be done by requiring a completely clean gap, i.e. without any soft hadrons. Indeed, all the QCD processes we consider are characterised by gluon (or quark) t-channel exchange, which unavoidably produces a colour flow along the gap. During hadronisation this colour flow, in turn, creates plenty of soft secondaries which fill the gap. On the other hand, there is no such effect for the electroweak graphs (Figs. 1, $2 \mathrm{a}, 2 \mathrm{~b})$ since the vector boson exchange is colourless. This means that if we require clean hadron-level gaps we can immediately discard the diagrams of Fig. 5. The only way to create a gap in a QCD induced event is to screen the colour flow (across the gap) by an additional gluon (or quark) exchange; that is, to consider graphs of the type shown in Fig. 19.

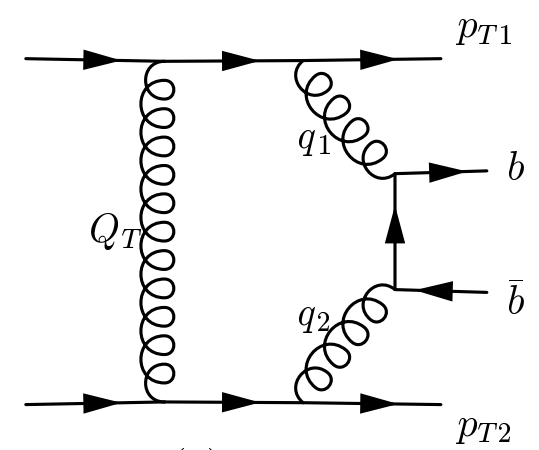

(a)

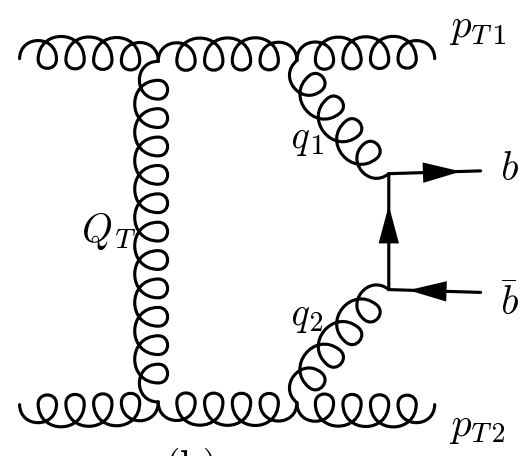

(b)

Figure 19: Screening of QCD dijet $+b \bar{b}$ production via gluon exchange.

Note that in leading order we can screen the colour flow in both gaps (above and below the $b \bar{b}$-pair) with only one additional $t$-channel gluon, with momentum $Q_{T}$ say.

\footnotetext{
${ }^{6}$ Note that the large survival probability $\hat{S}^{2} \sim 0.8$ used in [23] corresponds just to parton level gaps, and was calculated using the model of [35].
} 
The price we pay for this screening is a factor of $\alpha_{S}$ supplemented by the $d Q_{T}^{2}$ loop integration in each amplitude; that is $\left(\int \alpha_{S} \ldots d^{2} Q_{T}\right)^{2}$ in the cross section. At first sight, the major contribution comes from the small $Q_{T}$ region where the QCD coupling $\alpha_{S}$ is larger. Moreover, the integral takes the form

$$
\int \alpha_{s} \frac{d Q_{T}^{2}}{Q_{T}^{2}}
$$

and has infrared logarithmic divergence at $Q_{T} \ll p_{T j e t 1,2}$. However, this divergence is cut off by the effects of higher order double logarithmic QCD radiation, see for example \&8, 9 , 21]. The point is that a small $Q_{T}$ gluon screens the hard gluon at rather large distances $r \sim 1 / Q_{T}$ only. Thus a 'hard' gluon $q_{i=1,2}$ may emit a new 'semihard' gluon jet, with transverse energy $E_{T}$ ranging from $Q_{T}$ up to $q_{i T}=\left|\vec{p}_{T i}-\vec{Q}_{T}\right|$ in the whole rapidity gap interval $\Delta \eta_{i}$. The leading logarithms come from the $Q_{T} \ll E_{T} \ll p_{T j e t}$ domain where the expected mean number of these secondary gluons is

$$
\bar{n}_{i} \simeq \frac{N_{c} \alpha_{S}}{\pi} \Delta \eta_{i} \ln \frac{p_{T i}^{2}}{Q_{T}^{2}}
$$

At the amplitude level the corresponding suppression factor describing the probability for not having such an emission (which otherwise destroys the gap) has the Sudakov-like form

$$
\exp \left(-\bar{n}_{i} / 2\right)=\left(\frac{Q_{T}}{p_{T i}}\right)^{\frac{N_{c} \alpha_{S}}{2 \pi} \Delta \eta} .
$$

Including this factor in the loop integral, we eliminate the infrared divergence and obtain the probability, $P_{a}$ ( $a=q q, q g, g g$ depending on the initial state), to screen out the octet (gluon-like) colour flow in $q q$ ( $g g$ or $q g$ ) interactions,

$$
P_{a}=C_{a}\left(\int_{Q_{0}}^{p_{T \min }} \alpha_{S}\left(Q_{T}^{2}\right) \frac{d Q_{T}^{2}}{Q_{T}^{2}} \exp \left\{-\frac{N_{c} \Delta \eta}{2 \pi} \int_{Q_{T}}^{p_{T \min }} \alpha_{S}\left(Q^{\prime 2}\right) \frac{d Q^{\prime 2}}{Q^{\prime 2}}\right\}\right)^{2}=C_{a}\left(\frac{2 \pi}{N_{c} \Delta \eta}\right)^{2} .
$$

Here $\Delta \eta=\Delta \eta_{1}+\Delta \eta_{2}$ is the overall length of the gaps and, within leading logarithm accuracy, we have put the upper limits in the $Q_{T}\left(Q^{\prime}\right)$ integration equal to the minimum $p_{T}$ of the jets. In order to arrive at the right-hand side of Eq. (4.4) it is convenient to recast the integral in Eq. (4.4) as

$$
\left(\frac{2 \pi}{N_{c} \Delta \eta}\right) d \mathcal{J} \exp \left(-\mathcal{J}\left(p_{T \min }, Q_{T}\right)\right)
$$

with

$$
\mathcal{J}=\frac{N_{c} \Delta \eta}{2 \pi} \int_{Q_{T}}^{p_{T \min }} \alpha_{s}\left(Q^{\prime 2}\right) \frac{d Q^{\prime 2}}{Q^{\prime 2}}
$$


Performing the integration we neglect the term $\exp \left(-\mathcal{J}\left(p_{T \min }, Q_{0}\right)\right)$ corresponding to the lower limit of integration. This can always be done safely if we can continue the perturbative calculation down to the (rather low) scale where the quantity $\alpha_{s}\left(Q_{0}^{2}\right) \cdot \Delta \eta$ becomes large. Instead of the conventional double logarithm expressions (Eqs. (4.2) and (4.3)) with a fixed coupling $\alpha_{S}$, in Eq. (4.4) we have used the running coupling in order to demonstrate that the result does not depend on whether one accounts for the running $\alpha_{S}$ or not. The colour factors $C_{a}$ are

$$
C_{q q}=\frac{C_{F}^{2}}{\left(N_{c}^{2}-1\right)}=\frac{C_{F}}{2 N_{c}}=\frac{2}{9}, \quad C_{g g}=\frac{N_{c}^{2}}{N_{c}^{2}-1}=\frac{9}{8}, \quad C_{q g}=\frac{C_{F} N_{c}}{N_{c}^{2}-1}=\frac{1}{2} .
$$

A more precise way to calculate the contributions of Fig. 19 including QCD radiative effects is to replace the two gluon $t$-channel exchange by the non-forward BFKL amplitude [36]. For the asymmetric $\left(Q_{T} \ll q_{t i}\right)$ configuration the non-forward amplitude contains the double logarithmic factor of Eq. (14.3), while the single logarithmic $\left(\sim \mathcal{O}\left(\alpha_{s} \Delta \eta\right)\right)$ contribution in this asymmetric kinematical situation is suppressed, giving a less than $10 \%$ correction to the amplitude (see [21, 37] for a more detailed discussion). Thus we come back to the result of Eq. (4.4). Strictly speaking, besides the suppression factor Eq. (4.3) hidden in the BFKL amplitude, there should be another Sudakov-like double logarithmic form factor which reflects the absence of QCD radiation in the interval of gluon transverse momentum between $p_{T \text { jet }}$ and half of the boson (or $b \bar{b}$ ) mass, $M / 2$. However, in our case the transverse momentum of the jets is $p_{T}>p_{T \min }=40 \mathrm{GeV}$, which is close to half the boson mass $M_{Z, H} / 2$. Therefore the form factor becomes close to one and we can neglect it.

Another point we have to take into account is the fact that now the $b \bar{b}$-pair may be produced in a colour singlet state only, and the ordinary $g g \rightarrow b \bar{b}$ hard subprocess cross section (which includes both colour singlet and octet contributions) should be replaced by the pure colour singlet cross section 38

$\frac{1}{N_{c}^{2}-1} \frac{d \hat{\sigma}^{\text {incl }}}{d t}\left(g g^{P P} \rightarrow q \bar{q}\right)=\frac{\pi \alpha_{S}^{2}}{\left(N_{c}^{2}-1\right) E_{T}^{2} M^{2}} \frac{1}{6}\left[\left(1-\frac{2 E_{T}^{2}}{M^{2}}\right)\left(1-\frac{2 m_{q}^{2}}{E_{T}^{2}}\right)+\frac{m_{q}^{2}}{E_{T}^{2}}\left(1+\beta^{2}\right)\right]$,

where $\beta=\sqrt{1-\frac{4 m_{q}^{2}}{M^{2}}}$ and $m_{q}$ is the quark mass. Note that for the colour singlet production case there is an additional colour factor $1 /\left(N_{c}^{2}-1\right)$ which suppresses the QCD background, as the two colliding gluons are forced to have the same colour.

\subsection{Quark Exchange}

It is more difficult to screen the colour triplet flow originated by the quark exchange which we deal with in the electroweak and QCD $Z+2$ jet processes shown in Figs. 2 2 c, 


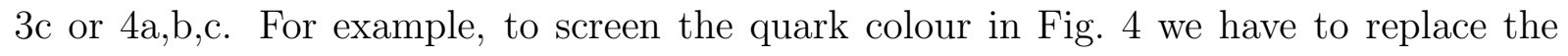
graphs Figs. 国a,b,c by those of Fig. 20. Due to the spin $1 / 2$ nature of a quark, the large

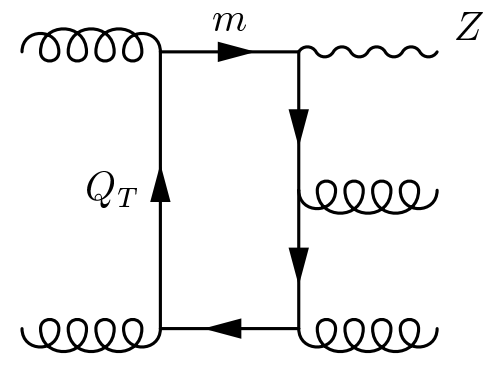

(a)

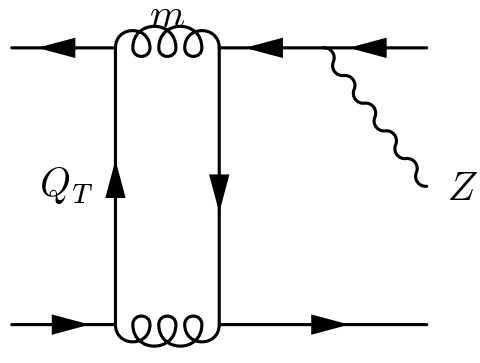

(b)

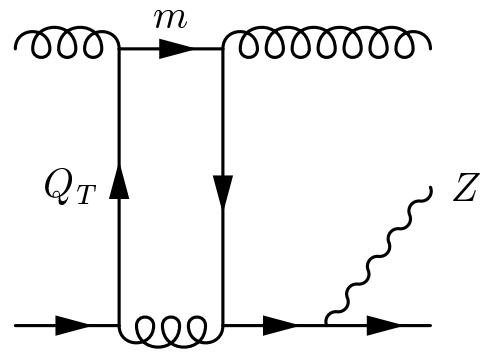

(c)

Figure 20: Screening of QCD dijet $+Z$ production via quark exchange.

rapidity gaps are suppressed at the amplitude level (in comparison with the corresponding Figs. Aa,b,c amplitude contribution) by the factor $e^{-\Delta \eta / 2}$ (i.e. a factor $e^{-\Delta \eta}$ in the cross section). On the other hand, it is known that the loop with two $t$-channel fermions may contain a double logarithm (see [39, 40]). One logarithm comes from the transverse $\left(Q_{T}\right)$ integration, while another logarithm (in the real part of the amplitude) originates from the $d m^{2} / m^{2}$ integral over the (virtual) mass of the upper $s$-channel particle in the loop (assuming that the contour of the Feynman integral is closed on the pole corresponding to the lower $s$-channel particle). In our kinematics, where a $Z$ boson is emitted in the centre of the rapidity gap interval, we obtain a logarithm when the mass, $m$, runs from $m^{2}=\max \left\{Q_{T}^{2}, \sqrt{\hat{s} M_{Z T}^{2}}\right\}$ up to $m^{2}=\hat{s}$ (here $\hat{s}$ is the incoming parton energy squared and $\left.M_{Z T}^{2}=M_{Z}^{2}+\left|p_{T Z}^{2}\right|\right)$. That is, the mass integral gives $\int \frac{d m^{2}}{m^{2}} \lesssim \frac{\Delta \eta}{2}$. The $Q_{T}$ integration does not give a logarithm in the case of Fig. 20a, but for the amplitudes corresponding to Fig. 20b,c, a logarithmic integral appears in the domain $p_{T \text { jet }}^{2} \ll Q_{T}^{2} \ll \hat{s}^{2} / 4$. Thus from the Fig. 20]b,c loop integrals we may expect a $\frac{3}{8}(\Delta \eta)^{2}$ enhancement. However, with our large rapidity gap $(\Delta \eta \simeq 6)$ the whole factor $-\left[\frac{\alpha_{S}}{\pi} \frac{3}{8}(\Delta \eta)^{2} e^{-\Delta \eta / 2}\right]^{2}=0.45\left(\frac{\alpha_{S}}{\pi}\right)^{2} \sim 10^{-3}$ is very small. Besides this, after the parton-level cuts described in Section 3.2 are applied, the original parton-level contribution of the diagrams with a ( $t$-channel) quark exchange is strongly suppressed. Therefore we neglect these contributions.

\subsection{Soft Survival Probability}

Returning to our original processes, we keep now only the graphs with either vector boson or two gluon (Fig. 19) exchange across the rapidity gaps, and multiply the corresponding parton-level cross sections by the appropriate gap survival probability $\hat{S}^{2}$. However, as 
we now require there to be no hadrons (even with a rather low $p_{T}$ ) in the gap interval, we have to take account of any soft interactions of the spectator partons.

Instead of using a Monte Carlo simulation, it is better to choose a model based on Regge (Pomeron) theory tuned to describe soft interaction data at high energies. We will use the model of Ref. [19]. This is based on the two-channel eikonal formalism, which reproduces all the main features of the soft $\left(\sigma_{t o t}, d \sigma_{e l} / d t\right)$ cross section data in the ISR-Tevatron energy range. Recall that the two channels of the eikonal correspond to two eigenstates which have different absorptive cross sections (i.e. different rescattering probabilities). Assuming the same (momentum and spatial) distributions of quarks and gluons in both components of the incoming proton wavefunction (that is, in both eigenstates of the eikonal) the model predicts for all our processes $\hat{S}^{2}=0.1$ at LHC energies. In other words, by requiring gaps at the hadron level we decrease the cross section by an order of magnitude. At first sight, the gap survival probability $\hat{S}^{2}=0.1$ reflects the rescattering of soft spectator partons and should, therefore, be universal for any process which has a gap[? However, this is not completely true. First, the value of $\hat{S}^{2}$ depends on the spatial distribution of parton spectators and therefore on the characteristic impact parameter $\left(b_{T}\right)$ difference between the two colliding protons [19, 20]. For example, in the case of exclusive Higgs boson production, $p p \rightarrow p+H+p$ via photon-photon fusion, the transverse momenta of the photons are very small. Hence the impact parameter $b_{T}$ is very large. The probability of soft rescattering in such a highly peripheral collision is small, and the value of $\hat{S}^{2}(\sim 0.9)$ is close to one [9, 11]. Secondly, there is a difference in the momentum distributions of partons in a different (diffractive eigenstate) component of the incoming proton wavefunction; it is reasonable to expect that the component with a smaller cross section contains more valence quarks (and 'hard' large- $x$ partons), whereas the component with a larger cross section has more low- $x$ gluons. This possibility was discussed in 20]. In such an approach, the model describes the breakdown of factorisation, in that there is about a factor 10 difference between the 'effective' Pomeron structure functions measured in diffractive deeply inelastic interactions at HERA and diffractive high- $E_{T}$ dijet hadroproduction at the Tevatron 26$]^{8}$.

In the present context, as the background $b \bar{b}$-pairs are produced predominantly in gluon-gluon collisions, the gap survival probability for the QCD background is a little smaller than for $Z(H)$-boson production via vector boson fusion where we deal with incoming quark-quark interactions (see Figs. 11 and 2). Using the formalism of Ref. [20]

\footnotetext{
${ }^{7}$ The only difference may be caused by the Sudakov-like form factor that accounts for the absence of QCD gluon bremsstrahlung in a specific hard subprocess.

${ }^{8}$ The difference is explained simply by the fact that the gap survival factor is $\hat{S}^{2} \sim 0.1$ for protonantiproton collisions, whereas $\hat{S}^{2} \simeq 1$ in deep inelastic scattering.
} 
we obtain for the kinematics (cuts) described in Section 3,

$$
\hat{S}_{Z}^{2}=0.31 ; \quad \hat{S}_{H}^{2}=0.31 ; \quad \hat{S}_{Q C D b \bar{b}}^{2}=0.27 .
$$

These survival factors are much larger than in the original model [19] because for the case considered here, of large rapidity gaps and large jet transverse momenta, we select mainly fast incoming partons and valence quarks which belong to the second component of the proton wavefunction. This component has a smaller absorptive cross section? In this case the QCD background is additionally suppressed 2.5 times. Note that both versions of the model [20] describe the diffractive dijet CDF data 26] well enough. On the other hand, in processes with large rapidity gaps at the LHC the uncertainty in the soft survival factor $\hat{S}^{2}$ may be rather large. It will therefore be important to study such a process experimentally. A promising way to study the survival probability $\hat{S}^{2}$ in different components of the incoming proton wave function (i.e. the dependence of $\hat{S}^{2}$ on the $p_{T \text { jet }}$ and rapidity cuts) is to measure QCD dijet production with rapidity gaps on either side of the dijet pair. Here the cross section is much larger (especially for gluon-gluon induced dijets) and it is easy to study the gap survival factor $\hat{S}^{2}$ under the various kinematic conditions: $p_{T}$ of the fast (large $\eta$ ) jets, size of the rapidity gaps, dijet mass, etc. In this way we can emphasise the rôle of the incoming valence quarks, sea quarks or gluons in different $x$ and scale $\mu^{2} \sim p_{T}^{2}$ domains, and hence choose the configuration where one or other component of the wavefunction dominates.

Note that, depending on the jet-finding algorithm, some soft hadrons may or may not be attributed to a particular $b$-jet. Therefore, one has to be more specific in the definition of the rapidity gap on the hadronic level in the presence of the high- $p_{T}$ jets. It looks plausible to select the gap by the requirement not to have hadrons within the gap range, apart from the cones of a fixed size $\Delta R \sim 1$ around the jet directions. In a real life experiment, jet-finding algorithms should be utilised in optimising the value of $\Delta R$. Soft survival factors $\hat{S}^{2}$ are practically independent of the $\Delta R$ value at $\Delta R \leq 2$.

\section{Results}

Fig. 21 shows the cross sections after hadronisation for central production of Higgs or $Z$ with rapidity gaps and subsequent decay to $b \bar{b}$ as a function of $p_{T \text { min }}$ of the proton

\footnotetext{
${ }^{9}$ Under the extreme hypothesis that all valence quarks belong to the second (low $\sigma_{a b s}$ ) component while gluons and sea quarks are concentrated in the first component (with a larger cross section) we get

$$
\hat{S}_{H}^{2}=\hat{S}_{Z}^{2}=0.26 \quad \text { and } \quad \hat{S}_{Q C D b \bar{b}}^{2}=0.10 .
$$
}




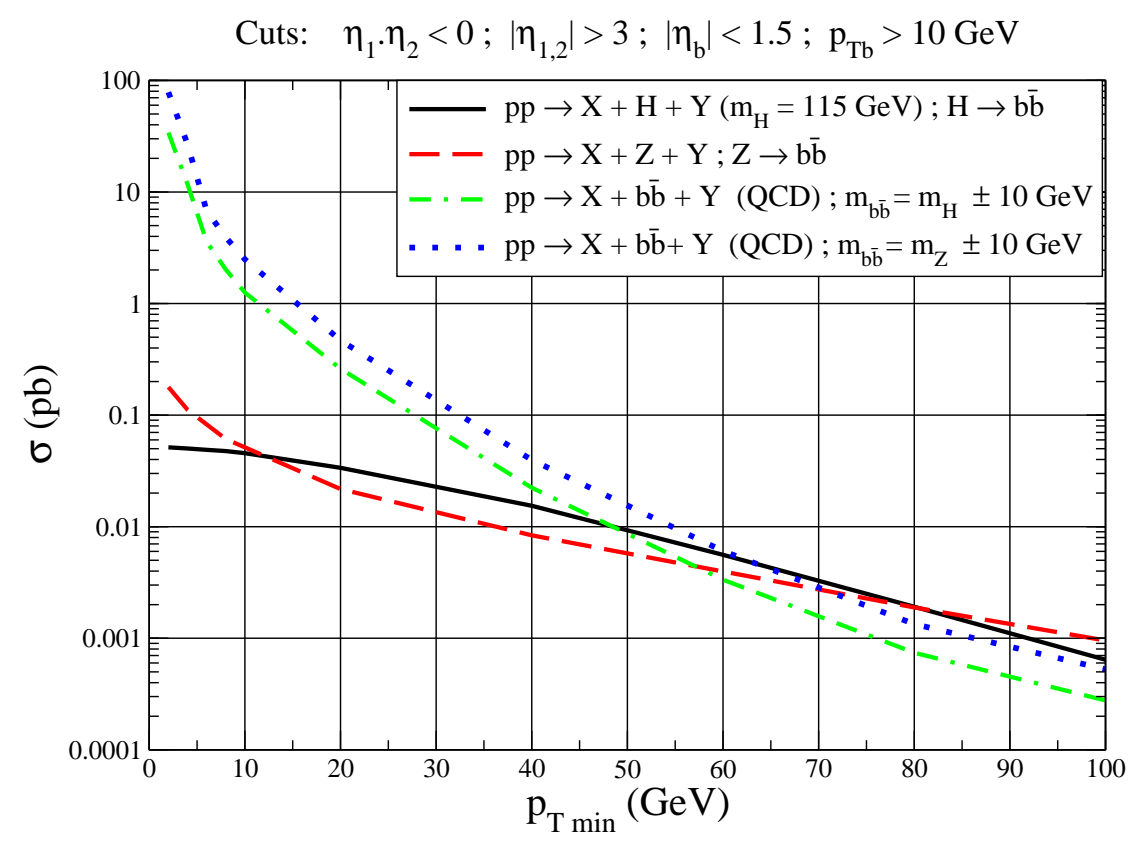

Figure 21: Hadron-level cross sections at $\sqrt{s}=14 \mathrm{TeV}$ for inclusive Higgs and $Z$ production with subsequent decay to $b \bar{b}$ and their respective QCD $b \bar{b}$ backgrounds. Cuts are applied at the parton level as discussed in the text.

remnant jets. It also shows the expected background of QCD $b \bar{b}$ events that display the same kinematical configuration. These are calculated using as a starting point the parton level cross sections after application of cuts, namely Figure 17 for Higgs production and Figure 18 for $Z$ production. The QCD-induced cross sections (both the QCD $Z$ production of Fig. $4 \mathrm{~d}$ and the direct QCD $b \bar{b}$ production of Fig. 5) are then multiplied by the probability to screen out the colour octet contribution for the relevant initial state of either $q q, q g$ or $g g$ (Eq. (4.4)). To take into account the fact that the $b \bar{b}$ pair in the background processes can only be produced in the colour singlet state the ordinary $g g \rightarrow b \bar{b}$ cross section is replaced by the pure singlet cross section, Eq. (4.8). Finally both the signals and backgrounds are multiplied by the relevant soft survival probability of Eq. (4.9).

We see that, as long as we stay away from the low $p_{T \text { min }}$ region, the signal for Higgs production is comparable with the QCD background, even exceeding it above $p_{T \min }=$ $50 \mathrm{GeV}$. The cross section for $Z$ production with rapidity gaps is less than that for Higgs production over most of the plot. This is because the branching fraction to $b \bar{b}$ is much lower than for the Higgs. Exceptions to this occur at low $p_{T \text { min }}$ where the effect of the infrared singularity makes its presence felt and at very high $p_{T \min }$, explained by the fact 
that the parton-level Higgs cross section falls more rapidly, as shown in Fig. 6. The backgrounds show an extremely strong dependence on $p_{T \text { min }}$, falling by five orders of magnitude as one varies $p_{T \text { min }}$ from $2 \mathrm{GeV}$ to $100 \mathrm{GeV}$. The QCD $b \bar{b}$ background with the invariant mass of the $b \bar{b}$ pair taken around the $Z$ mass is approximately $80 \%$ higher than that evaluated around the Higgs mass.

It should be noted that in these calculations we have taken $\alpha_{S} \equiv \alpha_{S}\left(M_{Z}^{2}\right)$. An argument could also be made that the characteristic scale should be that of the transverse momenta of the forward jets, i.e. $\alpha_{S} \equiv \alpha_{S}\left(p_{T \min }^{2}\right)$. This would affect the $\mathcal{O}\left(\alpha_{S}^{4}\right)$ backgrounds in such a way as to increase them by approximately $30 \%$ if we take the typical $p_{T}$ to be $40 \mathrm{GeV}$.

Let us emphasise that up to now we have not addressed the experimental issues. In particular, the predictions given in Fig. 21 should be modified to account for the $b$-tagging efficiency $\varepsilon_{b}$. This, in turn, is correlated with the probability $\mathcal{P}(g, q / b)$ to misidentify a gluon (or a light quark) jet as a $b$-jet. Recall that the rate of the $g g$-dijets exceeds the $b \bar{b}$-yield by two orders of magnitude. As discussed in [17], it is feasible to expect for the two $b$-jets $\left(\varepsilon_{b}\right)^{2}=0.6$ with $\mathcal{P}(g, q / b)=0.01$.

\section{Conclusions}

The weak boson fusion mechanism can provide a promising way to detect a light Higgs boson at the LHC, see for example [18, 22, 23]. The selection of events with large rapidity gaps and energetic large $p_{T}$ (quark) jets in the forward and backward directions allows the suppression of the QCD $b \bar{b}$ background down to a level comparable to the signal. Therefore, it becomes feasible to observe a light Higgs boson via its main $H \rightarrow b \bar{b}$ decay mode in addition to the usually discussed $\tau \tau$ and $W W^{*}$ channels, see for example [17].

The cross section for the production of a $115 \mathrm{GeV}$ Higgs boson in association with rapidity gaps at the LHC is expected to be about $15 \mathrm{fb}$ (for $p_{T}>40 \mathrm{GeV}$ ). Therefore, for an integrated luminosity of $30 \mathrm{fb}^{-1}$ planned for the first two or three years of LHC running, about 400 events can be observed.

Note that our cuts were not finally optimised for the particular ATLAS/CMS conditions. Thus, the significance of the signal may be improved by allowing asymmetric configurations with some minimal $\Delta \eta$ between the high- $p_{T}$ jets instead of the require-

ment $\left|\eta_{1,2}\right| \geq 3, \eta_{1} \cdot \eta_{2} \leq 0$. Such a kinematical choice was considered, for instance, in Ref. [18]. It is shown that this will noticeably improve the significance of the signal.

An important ingredient in the evaluation of both the signal and the background in 
the $b \bar{b}+2$ forward jet events is the soft survival factor $\hat{S}^{2}$, defining the probability that the gaps survive the soft $p p$-scattering. Recall that though this factor can be computed within the framework of existing models for soft rescattering, it is always unwise to rely on the precision of models based on soft physics. Fortunately, the soft survival factor for the gaps surrounding $W W \rightarrow H$ fusion can be monitored experimentally by observing the closely related central production of a $Z$ boson with the same rapidity gap and jet configuration [29, 30].

As was emphasised in [30, 23, 35], the $\tau \tau$ and $W W^{*}$ decay channels with rapidity gap kinematics can give a rather high significance for the observation of a light Higgs. In the $\tau \tau$ case the main background results from the tail in the $\tau \tau$ mass distribution generated by the $Z \rightarrow \tau^{+} \tau^{-}$decay. Again, the experimental observation of $Z$ boson central production allows one to control and monitor such a background.

It is worthwhile to mention that the experimental determination of the gap survival factor in the processes under consideration is interesting in its own right, since it provides important information on the incoming proton wavefunction. Note that since the incoming partons in the subprocess $q q \rightarrow q+(b \bar{b})+q$ are rather hard, the factor $\hat{S}^{2}$ depends on the model assumptions more sensitively than, for example, in the exclusive diffractive production case $p p \rightarrow p+b \bar{b}+p$, see [11, 19]. As was demonstrated in Section 4.4 (see footnote ${ }^{9}$ ) the results strongly depend on how the partons in the proton are distributed between the different diffractive eigenstates. Currently our information on these distributions is rather limited.

This paper concentrates on the detailed analysis of central $Z$ boson production accompanied by rapidity gaps on either side and two forward jets at the LHC. The QCD background processes for $Z+2$ jet production in the rapidity gap environment are addressed in detail. We evaluate the soft survival factors $\hat{S}^{2}$ for various processes under consideration. Finally, we note that it will be important to extend our work by incorporating a realistic Monte Carlo simulation, which will allow detector simulation to be included. We believe that the results presented in this paper make such an effort worthwhile.

\section{Acknowledgments}

We thank A. De Roeck, A. D. Martin and R. Orava for useful discussions. One of us (VAK) thanks the Leverhulme Trust for a Fellowship. This work was partially supported by the UK Particle Physics and Astronomy Research Council, by the Russian Fund for Fundamental Research (grants 01-02-17095 and 00-15-96610) and by the EU Framework 
TMR programme, contract FMRX-CT98-0194 (DG12-MIHT).

\section{References}

[1] D. Charlton, Experimental tests of the Standard Model arXiv:hep-ex/0110086. The LEP Electroweak Working Group, http://lepewwg.web.cern.ch/LEPEWWG.

[2] S. Heinemeyer, W. Hollik and G. Weiglein, Eur. Phys. J. C9, (1999) 343 arXiv:hep$\mathrm{ph} / 9812472]$.

[3] Yu. L. Dokshitzer, V. A. Khoze and S. I. Troyan, Sov. J. Nucl. Phys. 46, (1987) 712.

[4] Yu. L. Dokshitzer, V. A. Khoze and T. Sjöstrand, Phys. Lett. B274 (1992) 116.

[5] J. D. Bjorken, Int. J. Mod. Phys. A7 (1992) 4189; Phys. Rev. D47 (1993) 101.

[6] A. Bialas and P. V. Landshoff, Phys. Lett. B256 (1991) 540.

[7] R. S. Fletcher and T. Stelzer, Phys. Rev. D48 (1993) 5162 and references therein.

[8] V. A. Khoze, A. D. Martin and M. G. Ryskin, Phys. Lett. B401 (1997) 330.

[9] V. A. Khoze, A. D. Martin and M. G. Ryskin, Eur. Phys. J. C14 (2000) 525.

[10] E. M. Levin, arXiv:hep-ph/9912403 and references therein.

[11] V. A. Khoze, A. D. Martin and M. G. Ryskin, Eur. Phys. J. C23 (2002) 311 and references therein.

[12] M. Boonekamp, R. Peschanski and C. Royon, Phys. Rev. Lett. 87 (2001) 251806;

M. Boonekamp, A. De Roeck, R. Peschanski and C. Royon, arXiv:hep-ph/0205332 and references therein.

[13] B. E. Cox, J. R. Forshaw and B. Heinemann, arXiv:hep-ph/0110173;

R. B. Appleby and J. R. Forshaw, Phys. Lett. B541 (2002) 108 arXiv:hepph/0111077] and references therein.

[14] R. Enberg, G. Ingelman, A. Kissavos and N. Timneanu, arXiv:hep-ph/0203267 and references therein.

[15] V. A. Khoze, A. D. Martin and M. G. Ryskin, Eur. Phys. J. C19 (2001) 477, Erratum, ibid C20 (2001) 599. 
[16] V. A. Khoze, A. D. Martin and M. G. Ryskin, arXiv:hep-ph/0006005, in Proc. of 8th Int. Workshop on Deep Inelastic Scattering and QCD (DIS2000), Liverpool, eds J. Gracey and T. Greenshaw (World Scientific, 2001), p.592; V. A. Khoze, arXiv:hep$\mathrm{ph} / 0105224$.

[17] A. De Roeck, V. A. Khoze, A. D. Martin, R. Orava and M. G. Ryskin, arXiv:hep$\mathrm{ph} / 0207042$.

[18] V. A. Khoze, A. D. Martin and M. G. Ryskin, Eur. Phys. J. C21 (2001) 99.

[19] V. A. Khoze, A. D. Martin and M. G. Ryskin, Eur. Phys. J. C18 (2000) 167.

[20] A. B. Kaidalov, V. A. Khoze, A. D. Martin and M. G. Ryskin, Eur. Phys. J. C21 (2001) 521.

[21] A. D. Martin, V. A. Khoze and M. G. Ryskin, Phys. Rev. D56 (1997) 5867.

[22] D. Zeppenfeld, R. Kinnunen, A. Nikitenko and E. Richter-Was, Phys. Rev. D62 (2000) 013009.

[23] N. Kauer, T. Plehn, D. Rainwater and D. Zeppenfeld, Phys. Lett. B503 (2001) 113. D. Zeppenfeld, arXiv:hep-ph/0203123.

[24] E. Gotsman, E. Levin and U. Maor, Phys. Rev. D60 (1999) 094011 and references therein.

[25] M. M. Block and F. Halzen, Phys. Rev. D63 (2001) 114004.

[26] CDF Collaboration: T. Affolder et al., Phys. Rev. Lett. 84 (2000) 5043.

[27] R. Enberg, G. Ingelman and N. Timneanu, J. Phys. G26 (2000) 71 and references therein.

N. Timneanu and G. Ingelman, arXiv:hep-ph/0006227.

[28] A. Bialas, arXiv:hep-ph/0205059 and references therein.

[29] H. Chehime and D. Zeppenfeld, Phys. Rev. D47 (1993) 3898.

[30] D. Rainwater, R. Szalapski and D. Zeppenfeld, Phys. Rev. D54 (1996) 6680.

[31] T. Stelzer and W. F. Long, Comput. Phys. Commun. 81 (1994) 357 arXiv:hepph/9401258].

[32] V. A. Khoze, A. D. Martin and M. G. Ryskin, arXiv:hep-ph/0201301. 
[33] A. D. Martin, R. G. Roberts, W. J. Stirling and R. S. Thorne, Eur. Phys. J. C4 (1998) 463 [arXiv:hep-ph/9803445].

[34] T. Sjöstrand, Comput. Phys. Commun. 82 (1994) 74, http://www.thep.lu.se/torbjorn/Pythia.html. T .Sjöstrand et al., Comput. Phys. Commun. 135 (2002) 238

[35] D. Rainwater and D. Zeppenfeld Phys. Rev. D60 (1999) 113004, Erratum ibid. 61 (2000) 099901.

K. Hagiwara, D. Rainwater and D. Zeppenfeld, Phys. Rev. D59 (1999) 014037.

[36] L. N. Lipatov, Sov. Phys. JETP 63 (1986) 904.

[37] J. R. Forshaw, M. G. Ryskin, Z. Phys. C68 (1995) 137.

[38] V. A. Khoze, A. D. Martin and M. G. Ryskin, Eur. Phys. J. C23 (2002) 311 arXiv:hep-ph/0111078.

[39] V. G. Gorshkov, V. N. Gribov, L. N. Lipatov and G. V. Frolov, Sov. J. Nucl. Phys. 6 (1967) 95.

[40] R. Kirschner, L. N. Lipatov, Nucl. Phys. B213 (1983) 122. 